\title{
FREE SYMMETRIC AND UNITARY PAIRS IN THE FIELD OF FRACTIONS OF TORSION-FREE NILPOTENT GROUP ALGEBRAS
}

\author{
VITOR O. FERREIRA, JAIRO Z. GONÇALVES, AND JAVIER SÁNCHEZ
}

\begin{abstract}
Let $k$ be a field of characteristic different from 2 and let $G$ be a nonabelian residually torsion-free nilpotent group. It is known that $G$ is an orderable group. Let $k(G)$ denote the subdivision ring of the Malcev-Neumann series ring generated by the group algebra of $G$ over $k$. If $*$ is an involution on $G$, then it extends to a unique $k$-involution on $k(G)$. We show that $k(G)$ contains pairs of symmetric elements with respect to $*$ which generate a free group inside the multiplicative group of $k(G)$. Free unitary pairs also exist if $G$ is torsion-free nilpotent. Finally, we consider the general case of a division ring $D$, with a $k$-involution $*$, containing a normal subgroup $N$ in its multiplicative group, such that $G \subseteq N$, with $G$ a nilpotent-by-finite torsion-free subgroup that is not abelian-by-finite, satisfying $G^{*}=G$ and $N^{*}=N$. We prove that $N$ contains a free symmetric pair.
\end{abstract}

\section{INTRODUCTION}

In [13, Lichtman raises the interesting

Conjecture 1.1. The multiplicative group of a noncommutative division ring contains a free noncyclic subgroup.

Frequently division rings come accompanied by involutions. One instance is the case of division rings of fractions of group algebras which are Ore domains.

To be more precise, by an involution on a group $G$ one understands an anti-automorphism of $G$ of order 2. If $k$ is a field a $k$-involution on a $k$-algebra $R$ is a $k$-linear map $*$ satisfying $(x y)^{*}=y^{*} x^{*}$ and $\left(x^{*}\right)^{*}=x$ for all $x, y \in R$. Thus, if $G$ is a group and $k$ is a field, the $k$-linear extension of an involution on $G$ to the group algebra $k[G]$ is a $k$-involution. Suppose that $k[G]$ is embedded in a division ring $D$ generated (as a division ring) by $k[G]$. There is at most one involution on $D$ which is the extension of the involution on $k[G]$. When this is the case, we shall say that the $k$-involution on $D$ is induced by the involution of $G$. For example, if $k[G]$ is an Ore domain with division ring of fractions $D$, there always exists a $k$-involution on $D$ induced by the involution of $G$.

An element $x$ of an algebra $R$ with an involution $*$ is called symmetric if $x^{*}=x$ and unitary if $x x^{*}=x^{*} x=1$. And if $x$ and $y$ are elements of $R$, we say that they form a free symmetric (resp. unitary) pair if both are symmetric (resp. unitary) and generate a free noncyclic subgroup of the group of units of $R$. We shall look into the existence of free symmetric and unitary pairs in division rings with involution. In what follows, if $D$ is a division ring, we shall denote the multiplicative group $D \backslash\{0\}$ of $D$ by $D^{\bullet}$.

Date: 21 May 2018.

2010 Mathematics Subject Classification. Primary 16K40, 16W10, 16S85, 16W60; Secondary 20F18, 20 F60.

Key words and phrases. Infinite dimensional division rings, division rings with involution, (residually) torsion-free nilpotent groups.

The first author was partially supported by FAPESP-Brazil, Proj. Temático 2015/09162-9.

The second author was partially supported by FAPESP-Brazil, Proj. Temático 2015/09162-9, and CNPq, Grant 301205/2015-9.

The third author was partially supported by FAPESP-Brazil, Proj. Temático 2015/09162-9, and CNPq, Grant $307638 / 2015-4$. 
In [8], an analogue of Conjecture 1.1 was proved for finite-dimensional division rings with involutions, namely: let $D$ be a division algebra over a field $k$ of characteristic different from 2 and let * be a $k$-involution on $D$. If $D$ is finite dimensional over its center, then it contains free symmetric and unitary pairs, unless it is a quaternion algebra with an appropriate involution in each case. In [1, 9] and [4, the existence of free symmetric and unitary pairs when $D$ is a division ring with involution, infinite-dimensional over its center, $k$ was considered.

In particular, in [4], it was shown that for the division ring of fractions of the group algebra $k[\Gamma]$ of the Heisenberg group

$$
\Gamma=\langle x, y:[x,[x, y]]=[y,[x, y]]=1\rangle,
$$

where $[x, y]$ stands for the commutator $x^{-1} y^{-1} x y$, the following holds.

Theorem 1.2 ([4, Theorem 2]). Let $D$ be the division ring of fractions of the group algebra $k[\Gamma]$ of the Heisenberg group $\Gamma$ over a field $k$ of characteristic different from 2 , and let $*$ be a $k$-involution of $D$ induced from an involution of $\Gamma$. Then $D$ contains both free symmetric and free unitary pairs with respect to $*$.

Our objective in Section 2 is, following the ideas of [1, to present a proof of

Theorem 1.3. Let $k[G]$ be the group algebra of a nonabelian torsion-free nilpotent group $G$ over a field $k$ of characteristic different from 2 and let $D$ be the division ring of fractions of $k[G]$. Let $*$ be a $k$-involution on $D$ which is induced by an involution of $G$. Then $D$ contains both free symmetric and free unitary pairs with respect to $*$.

Regarding the existence of free symmetric pairs, we can also handle the case of normal subgroups of division rings containing a torsion-free nilpotent-by-finite subgroup not abelian-by-finite, as the following result shows. We note that, by [12, 1.3.2], the class of nilpotent-by-finite groups contains the class of supersoluble groups.

Theorem 1.4. Let $D$ be a division ring with center $k$ of characteristic different from 2 , with a $k$-involution *. Let $N$ be a normal subgroup of $D^{\bullet}$ and assume that $N$ contains a torsion-free nilpotent-by-finite subgroup $G$ which is not abelian-by-finite. If both $G$ and $N$ are invariant under $*$, then $N$ contains a free symmetric pair with respect to $*$.

One can say that Theorem 1.4 is an involutional version, for symmetric elements, of the following result of Lichtman.

Theorem 1.5 ([14, Theorem 2]). Let $D$ be a division ring and let $N$ be a normal subgroup of $D^{\bullet}$. Suppose that there exists a nonabelian nilpotent-by-finite subgroup $G$ of $N$. Then $N$ contains a noncyclic free subgroup.

Unfortunately, we do not have a complete analogue of this result, since we are still unable to handle the case $N \triangleleft D^{\bullet}$ when $D$ is finite-dimensional over its center $k$. This happens precisely when $G$ is abelian-by-finite.

The restriction on the characteristic of $k$ is usual, since many strange things happen for involutions when the characteristic is 2 . For instance, our technique to construct specializations from the division ring of fractions of the Heisenberg group algebra to a quaternion algebra collapses in characteristic 2 .

Section 3 presents a completion argument that allows the main result on free symmetric pairs of Section 2 to be extended to residually torsion-free nilpotent groups. More precisely, given a field $k$, a residually torsion-free nilpotent group $G$ and a total ordering $<$ on $G$ such that $(G,<)$ is an ordered group, let $k(G)$ denote the subdivision ring of the Malcev-Neumann series $\operatorname{ring} k((G,<))$ generated by the group algebra $k[G]$. Let $*$ be a $k$-involution on $G$ which is induced by an involution of $G$. In [2, Theorem 2.9], it was proved that $*$ has a unique extension to a $k$-involution of $k(G)$. The main result of Section 3 is the following. 
Theorem 1.6. Let $k$ be a field of characteristic different from 2 and let $G$ be a nonabelian residually torsion-free nilpotent group with an involution $*$. Then $k(G)$ contains a free symmetric pair with respect to the $k$-involution induced by $*$.

\section{TORSION-FREE NILPOTENT GROUPS}

2.1. Auxiliary results. The proofs of Theorems 1.3 and 1.4 are obtained constructing specializations from the division ring of fractions of a skew polynomial ring to a division ring known to contain free subgroups.

The theory we use are detailed in [7] and will be briefly recalled below.

By a specialization from a division ring $D$ to a division ring $E$, we mean an epimorphism $\psi: T \rightarrow$ $E$ from a subring $T$ of $D$ such that every element of $T$ not in $\operatorname{ker} \psi$ is invertible in $T$. In particular, a pair of elements of $T$ mapping to a pair in $D$ which generates a free subgroup in $E^{\bullet}$ will generate a free subgroup in $D^{\bullet}$.

We shall be considering specializations to symbol and quaternion algebras, whose definitions we recall.

Let $m$ be a positive integer which is invertible in $k$ and suppose that $k$ contains a primitive $m$-th root of unity $\theta$. Let $a, b \in k^{\bullet}$. The symbol algebra $\mathcal{S}_{k}(a, b, \theta)$ is the $k$-algebra generated by $\mathbf{i}$ and $\mathbf{j}$ subject to

$$
\mathbf{i}^{m}=a, \quad \mathbf{j}^{m}=b, \quad \mathbf{j i}=\theta \mathbf{i j} .
$$

The algebra $S_{k}(a, b, \theta)$ is central simple over $k$ of dimension $m^{2}$ with basis $\left\{\mathbf{i}^{r} \mathbf{j}^{s}: 0 \leq r, s \leq m-1\right\}$. In the case $m=2$, we get the so called quaternion algebras. Therefore, the quaternion algebra $\mathcal{S}_{k}(a, b,-1)$ over a field $k$ of characteristic $\neq 2$ is the 4 -dimensional $k$-algebra with basis $\{1, \mathbf{i}, \mathbf{j}, \mathbf{k}\}$ with multiplication satisfying

$$
\mathbf{i}^{2}=a, \quad \mathbf{j}^{2}=b, \quad \mathbf{j i}=-\mathbf{i} \mathbf{j}=-\mathbf{k} .
$$

Some symbol algebras are division rings. This will be the case in Proposition 2.1 below.

Let $P$ be a prime field, that is, $P=\mathbb{Q}$, the field of rational number, or $P=\mathbb{F}_{p}$, the field with $p$ elements for a prime number $p$, and let $P(\lambda, X)$ be the rational function field in the commuting indeterminates $\lambda$ and $X$ over $P$. Let $\sigma$ be the $P$-automorphism of $P(\lambda, X)$ such that $\sigma(\lambda)=\lambda$ and $\sigma(X)=\lambda X$, and consider the skew polynomial ring $P(\lambda, X)[Y ; \sigma]$ formed by skew polynomials of the form $c_{0}+c_{1} Y+\cdots+c_{n} Y^{n}$, with $c_{i} \in P(\lambda, X)$, satisfying the commutation rule $Y c=\sigma(c) Y$, for all $c \in P(\lambda, X)$.

Our aim is to construct a specialization from the division ring of fractions $D$ of $P(\lambda, X)[Y ; \sigma]$ to the symbol algebra $\mathcal{S}_{F}(a, b, \theta)$, where $q>1$ is an integer not divisible by $p, \theta$ is a primitive $q$-th root of unity and $F$ is the rational function field $P(\theta)(a, b)$ in the commuting indeterminates $a$ and $b$ over $P(\theta)$.

We define the domain of the specialization as follows. Let $f(\lambda)$ be the minimal polynomial of $\theta$ over $P$. The principal ideal $I=f(\lambda) P[\lambda]$ of $P[\lambda]$ is the kernel of the evaluation map $P[\lambda] \rightarrow$ $\mathcal{S}_{F}(a, b, \theta), \lambda \mapsto \theta$. This homomorphism can be extended in a unique way to a homomorphism $\phi: R \rightarrow \mathcal{S}_{F}(a, b, \theta)$, where $R$ stands for the localization of $P[\lambda]$ at $I$. Notice that the image of $\phi$ is contained in $P(\theta)$. Now, let $\varphi: R[X] \rightarrow \mathcal{S}_{F}(a, b, \theta)$ be the unique homomorphism coinciding with $\phi$ on $R$ such that $X \mapsto \mathbf{i}$. It is clear that the image of $\varphi$ is contained in $P(\theta)(\mathbf{i})$. Because the powers of $\mathbf{i}$ are left $P(\theta)$-linearly independent, one can see that $\operatorname{ker} \phi=f(\lambda) R[X]$. Now, since $Y X=\lambda X Y$ in $R[X][Y ; \sigma]$, there exists a unique extension of $\phi$ to a homomorphism $\psi: R[X][Y ; \sigma] \rightarrow \mathcal{S}_{F}(a, b, \theta)$ sending $Y \mapsto \mathbf{j}$. We claim that $\operatorname{ker} \psi$ coincides with the ideal of $R[X][Y ; \sigma]$ generated by the central element $f(\lambda)$. Indeed, given $W \in \operatorname{ker} \psi$, since the powers of $\mathbf{j}$ are left $P(\theta)(\mathbf{i})$-linearly independent, as a polynomial in $Y, W$ must have all its coefficients in $\operatorname{ker} \varphi$. It follows that $W$ is a multiple of $f(\lambda)$, as desired. Note that $\operatorname{ker} \psi$ is a completely prime ideal of $R[X][Y ; \sigma]$ and that $\bigcap_{n=0}^{\infty}(\operatorname{ker} \psi)^{n}=0$. Now [7, Lemma 3.2] implies that $M \backslash \operatorname{ker} \psi$ is a right denominator set in 
$R[X][Y ; \sigma]$. The localization of $R[X][Y ; \sigma]$ at $M$ is a local subring $T$ of $D$ and $\psi$ can be extended to $T$. Moreover, if $B$ is the maximal ideal of $T$ (and the kernel of $\psi$ ), then $T / B \cong \mathcal{S}_{F}(a, b, \theta)$. So $\psi$ is the desired specialization.

Now, $D$ is isomorphic to the division ring of fractions of the group algebra $P[\Gamma]$ of the Heisenberg group $\Gamma$ over $P$ under a map satisfying $\lambda \mapsto[y, x], X \mapsto x, Y \mapsto y$. Therefore, one obtains the following

Proposition 2.1. Let $P$ be a prime field, let $q \geq 1$ be an integer not divisible by the characteristic of $P$ and let $\theta$ be a primitive $q$-th root of unity. Let $D$ be the division ring of fractions of the group algebra $P[\Gamma]$ of the Heisenberg group $\Gamma$ over $P$. Then there exists a specialization $\psi$ from $D$ to $\mathcal{S}_{F}(a, b, \theta)$ such that $\psi(x)=\mathbf{i}, \psi(y)=\mathbf{j}$ and $\psi([y, x])=\theta$, where $F$ stands for the rational function field $P(\theta)(a, b)$ in the commuting indeterminates $a$ and $b$ over $P(\theta)$.

Our next step is to find inside a nonabelian torsion-free nilpotent group with an involution an invariant Heisenberg subgroup.

We shall accomplish this in Proposition 2.4.

Lemma 2.2. Let $G$ be a torsion-free nilpotent group with center $C$. If $G$ has an abelian subgroup $H$, containing $C$, such that for every $x \in G$, there exist a positive integer $r$ such that $x^{r} C \in H / C$, then $G$ is abelian.

Proof. Given $x, y \in G$, let $r, s$ be positive integers such that $x^{r} C$ and $y^{s} C$ belong to $H / C$. Since $H$ is abelian, it follows that $x^{r} y^{s}=y^{s} x^{r}$. But in a torsion-free nilpotent group this implies $x y=y x$ (see, e.g. [11, 16.2.9]).

We start by dealing with nilpotency class 2 . Recall that in a torsion-free group, any two elements which do not commute, whose commutator commute with both, generate a Heisenberg subgroup. In what follows, we shall frequently use the fact that if $G$ is a torsion-free nilpotent group with center $C$, then $G / C$ is torsion-free (for a proof, see [17, Lemma 11.1.3]).

Lemma 2.3. Let $G$ be a finitely generated torsion-free nilpotent group of class 2 with involution *. Then $G$ contains a *-invariant Heisenberg subgroup. More precisely, there exist $x, y \in G$ such that $[x, y] \neq 1, x^{*}=x^{ \pm 1}, y^{*}=y^{ \pm 1}$.

Proof. Let $C$ denote the center of $G$. It follows from the hypothesis on the nilpotency class of $G$ that $G / C$ is a finitely generated torsion-free abelian group, so there exists an isomorphism $\varphi: G / C \rightarrow \mathbb{Z}^{n}$, for some integer $n \geq 2$ (since $G$ is nonabelian). Because $C$ is a characteristic subgroup of $G$, it is invariant under $*$; so $*$ induces an automorphism on $G / C$ whose square is the identity. Therefore, there exists $A \in \mathrm{GL}(n, \mathbb{Z})$ such that $A^{2}=I_{n}$ and $\varphi\left(g^{*} C\right)=A \varphi(g C)$, for every $g \in G$.

Since $A$ is diagonalizable over $\mathbb{Q}$ with eigenvalues 1 or -1 , there exists a $\mathbb{Q}$-basis $\left\{v_{1}, \ldots, v_{n}\right\}$ of $\mathbb{Q}^{n}$ with $v_{i} \in \mathbb{Z}^{n}$ satisfying $A v_{i}= \pm v_{i}$, for all $i=1, \ldots, n$. For each $i=1, \ldots, n$, pick $h_{i} \in G$ such that $\varphi\left(h_{i} C\right)=v_{i}$ and let $H$ be the subgroup of $G$ generated by $\left\{h_{1}, \ldots, h_{n}\right\}$.

Given $x \in G$, let $q_{1}, \ldots, q_{n} \in \mathbb{Q}$ be such that $\varphi(x C)=q_{1} v_{1}+\cdots+q_{n} v_{n}$. Choose a positive integer $r$ such that $r q_{i}=a_{i} \in \mathbb{Z}$ for all $i=1, \ldots, n$. It follows that $x^{r} C=h_{1}^{a_{1}} \cdots h_{n}^{a_{n}} C \in H / C$. Since $G$ is nonabelian, it follows from Lemma 2.2, that $H$ is nonabelian; so, we can suppose that $\left[h_{1}, h_{2}\right] \neq 1$.

By the choice of these elements, it follows that there exist $z_{1}, z_{2} \in C$ such that $h_{i}^{*}=h_{i}^{\varepsilon_{i}} z_{i}$, where $\varepsilon_{i} \in\{-1,1\}$, for $i=1,2$. Moreover, these central elements satisfy $z_{i}^{*}=z_{i}^{-\varepsilon_{i}}$, since $h_{i}=\left(h_{i}^{*}\right)^{*}=$ $\left(h_{i}^{\varepsilon} z_{i}\right)^{*}=z_{i}^{*}\left(h_{i}^{*}\right)^{\varepsilon_{i}}=z_{i}^{*}\left(h_{i}^{\varepsilon_{i}} z_{i}\right)^{\varepsilon_{i}}=z_{i}^{*} z_{i}^{\varepsilon_{i}} h_{i}$.

We are now in a position to define the generators of a $*$-invariant subgroup of $G$. 
Let $x=h_{1}^{2 \varepsilon_{1}} z_{1}$ and $y=h_{2}^{2 \varepsilon_{2}} z_{2}$. Then

$$
\begin{aligned}
x^{*} & =\left(h_{1}^{2 \varepsilon_{1}} z_{1}\right)^{*}=z_{1}^{*}\left(h_{1}^{*}\right)^{2 \varepsilon_{1}}=z_{1}^{-\varepsilon_{1}}\left(h_{1}^{\varepsilon_{1}} z_{1}\right)^{2 \varepsilon_{1}}=z_{1}^{-\varepsilon_{1}} z_{1}^{2 \varepsilon_{1}} h_{1}^{2 \varepsilon_{1}^{2}} \\
& =z_{1}^{\varepsilon_{1}} h_{1}^{2}=\left(h_{1}^{2 \varepsilon_{1}} z_{1}\right)^{\varepsilon_{1}}=x^{\varepsilon_{1}}
\end{aligned}
$$

And similarly, $y^{*}=y^{\varepsilon_{2}}$.

It remains to prove that $x$ and $y$ do not commute. For that, we shall use the fact that in a nilpotent group of class 2, the commutator is a homomorphism in each entry (see [17, Lemma 3.4.1]). So, one has $[x, y]=\left[h_{1}^{2 \varepsilon_{1}} z_{1}, h_{2}^{2 \varepsilon_{2}} z_{2}\right]=\left[h_{1}, h_{2}\right]^{4 \varepsilon_{1} \varepsilon_{2}} \neq 1$, for $G$ is torsion-free.

Proposition 2.4. Let $G$ be a nonabelian torsion-free nilpotent group with involution *. Then $G$ contains a $*$-invariant Heisenberg subgroup. More precisely, there exist $x, y \in G$ such that $[x, y] \neq 1$, $[x,[x, y]]=[y,[x, y]]=1, x^{*}=x^{ \pm 1}, y^{*}=y^{ \pm 1}$.

Proof. By taking the subgroup of $G$ generated by two noncommuting elements and their images under $*$, we can assume that $G$ is finitely generated.

We shall argue by induction on the nilpotency class $c$ of $G$; the case $c=2$ having been dealt with in Lemma 2.3.

Suppose $c>2$ and let $C$ denote the center of $G$. Then $G / C$ is a nonabelian finitely generated torsion-free nilpotent group of class $c-1$, with an involution $*$ induced by $*$. By the induction hypothesis, there exist $x, y \in G$ such that $x^{*} C=x^{\varepsilon} C, y^{*} C=y^{\eta} C$, with $\varepsilon, \eta \in\{-1,1\}$, satisfying $z=[x, y] \notin C$ and $[x, z],[y, z] \in C$. It follows that $L=\langle x, y, C\rangle$ is a $*$-invariant subgroup of $G$ of class $\leq 3$.

If $L$ has class 2 , then the result follows from Lemma 2.3 .

Suppose that $L$ has class 3 . Then $[x, z] \neq 1$ or $[y, z] \neq 1$. Say $[x, z] \neq 1$. We shall show that $K=\left\langle x, x^{*}, z, z^{*}\right\rangle$ is a $*$-invariant subgroup of $G$ of class 2 . It will be enough to show that $[\alpha, \beta]$ lies in the center of $K$ for all $\alpha, \beta \in\left\{x, x^{*}, z, z^{*}\right\}$. For each $n \geq 1$, let $\gamma_{n}(L)$ denote the $n$-th term in the lower central series of $L$. Now, $z=[x, y] \in \gamma_{2}(L)$, so $z^{*} \in \gamma_{2}(L)$, because the terms in the lower central series are fully invariant subgroups of $G$. It follows that for every $\alpha \in K$ and $\beta \in\left\{z, z^{*}\right\}$ we have $[\alpha, \beta] \in \gamma_{3}(L)$, which is a central subgroup of $L$, hence $[\alpha, \beta]$ is central in $K$. Finally, that $\left[x, x^{*}\right]=1$ follows from the fact that $x^{*} x^{-\varepsilon} \in C$. So $K$ is indeed a $*$-invariant subgroup of $G$ of class 2, hence, Lemma 2.3 applies to it.

2.2. Proof of Theorem 1.3. By Proposition 2.4 $G$ contains elements $x, y$ such that $z=[y, x] \neq$ $1,[x, z]=[y, z]=1$, satisfying one of the following conditions

(1) $x^{*}=x, y^{*}=y$; so, clearly, $z^{*}=z^{-1}$;

(2) $x^{*}=x^{-1}, y^{*}=y^{-1}$; hence $z^{*}=z^{-1}$;

(3) $x^{*}=x, y^{*}=y^{-1}$ and $z^{*}=z$.

Let $P$ be the prime subfield of $k$ and let $D^{\prime}$ be the subdivision ring of $D$ generated by $x$ and $y$ over $P$. Then $D^{\prime}$ is the division ring of fractions of the group algebra $P[\Gamma]$, where $\Gamma$ is the subgroup of $G$ generated by $x$ and $y$, which is a Heisenberg group. The involution $*$ of $G$ restricts to an involution $*$ on $\Gamma$. So, we could use Theorem 1.2 to guarantee that $D^{\prime}$ and, a fortiori, $D$ contain free symmetric and unitary pairs.

However a simpler argument than the one used in [4] is available here. So we reprove the fact that $D^{\prime}$ contains both free symmetric and unitary pairs. We shall make use of Proposition 2.1 and use the notation $\mathcal{S}_{q}$ for the symbol algebra $\mathcal{S}_{F}(a, b, \theta)$ and $\mathcal{H}$ for the quaternion algebra $\mathcal{S}_{2}$.

We consider each of the three possibilities for $*$ on the generators $x$ and $y$ of $\Gamma$ at a time.

(1) $x^{*}=x, y^{*}=y, z^{*}=z^{-1}$. 
- Free symmetric pairs. Set $u=1+x, v=1+y$, and let $\psi$ be the specialization from $D^{\prime}$ to the quaternion algebra $\mathcal{H}$ provided by Proposition 2.1 that is, $\psi$ satisfies $\psi(x)=\mathbf{i}, \psi(y)=\mathbf{j}$ and $\psi(z)=-1$. Then $\psi(u)=1+\mathbf{i}, \psi(v)=1+\mathbf{j}$, and, by [6, Theorem 2], $\{u, v\}$ is a free symmetric pair.

- Free unitary pairs. Let $q>1$ be an integer not divisible by the characteristic of $P$ and let $\psi$ be the specialization from $D^{\prime}$ to $\mathcal{S}_{q}$ from Proposition 2.1. So we have $\psi(x)=\mathbf{i}$, $\psi(y)=\mathbf{j}$ and $\psi(z)=\theta$ and the relations $\mathbf{i}^{q}=a, \mathbf{j}^{q}=b, \mathbf{j i}=\theta \mathbf{i j}, \theta^{q}=1$ hold in $\mathcal{S}_{q}$. Set

$$
u=(1-z x)\left(1-z^{-1} x\right)^{-1} \text { and } v=(1-z y)\left(1-z^{-1} y\right)^{-1} .
$$

So, $\psi(u)=\frac{1-\theta \mathbf{i}}{1-\theta^{q-1} \mathbf{i}}$ and $\psi(v)=\frac{1-\theta \mathbf{j}}{1-\theta^{q-1} \mathbf{j}}$. Using the right regular representation with respect to the basis $\left\{1, \mathbf{j}, \ldots, \mathbf{j}^{q-1}\right\}$ of $\mathcal{S}_{q}$ over the field $P(\theta, a, b, \mathbf{i})$, we write $\psi(u)$ and $\psi(v)$ as matrices $A$ and $B$, respectively. Let $S$ be the ring of integers of $P(\theta, a, b)(\mathbf{i})$ over $P(\theta, b)[a]$. If $\nu$ denotes the nonarchimedean valuation determined by the maximal ideal of $S$ containing $1-\theta \mathbf{i}$, it is easy to verify that $A$ and $B$ satisfy the conditions of [5, Proposition 2.4]. Therefore $A$ and $B^{-1} A B$ generate a free subgroup, and so $\left\{u, v^{-1} u v\right\}$ is a free unitary pair in $D^{\prime}$.

(2) $x^{*}=x^{-1}, y^{*}=y^{-1}, z^{*}=z^{-1}$.

Use the same pairs as in the proof of [1, Theorem 1.1].

(3) $x^{*}=x, y^{*}=y^{-1}, z^{*}=z$.

Let $\psi$ be the specialization from $D^{\prime}$ onto the quaternion algebra $\mathcal{H}$ such that $\psi(x)=$ $\mathbf{i}, \psi(y)=\mathbf{j}, \psi(z)=-1$, afforded by Proposition 2.1.

- Free symmetric pairs. Set $u=1+x, r=x y^{5}-y^{-5} x$ and $v=(1-r)(1+r)^{-1}$. Then $u^{*}=u, r^{*}=-r$ and $v^{*}=v^{-1}$. Arguing as in the proof of [1, Theorem 1.1] for the existence of free symmetric pair, case (III)(i), we conclude that $\left\{u, v^{-1} u v\right\}$ is a free symmetric pair.

- Free unitary pairs. Set $u=x y^{5}-y^{-5} x, v=y-y^{-1}, r=(1-v)(1+v)^{-1}$ and $s=(1-u)(1+u)^{-1}$. Arguing as in the proof of [1, Theorem 1.1] for the existence of free unitary pairs, case (III)(i), we can show that $\psi(r)$ and $\psi\left(s^{-1} r s\right)$ generate a free subgroup. Therefore $\left\{r, s^{-1} r s\right\}$ is a free unitary pair.

2.3. Proof of Theorem 1.4. Since $G$ is a torsion-free nilpotent-by-finite group such that $G^{*}=G$, it follows that $G$ contains a nilpotent subgroup $H$ such that $[G: H]<\infty$. Let $H^{*}$ denote the image of $H$ under $*$ inside $G$. Since $\left[G: H^{*}\right]<\infty$, it follows, by Poincaré's theorem on intersections of subgroups of finite index, that $\left[G: H \cap H^{*}\right]<\infty$. But $G$ is not abelian-by-finite, so $H \cap H^{*}$ is nilpotent, nonabelian and invariant under *. Therefore, substituting $G$ by $H \cap H^{*}$, if necessary, we can assume that $G$ is nonabelian, torsion-free, nilpotent and invariant under $*$.

By Proposition 2.4, $G$ contains a $*$-invariant Heisenberg subgroup $\Gamma=\langle x, y, z:[y, x]=z \neq$ $1,[x, z]=[y, z]=1\rangle$ in which one of the following holds:

(1) $x^{*}=x, y^{*}=y$ and $z^{*}=z^{-1}$;

(2) $x^{*}=x, y^{*}=y^{-1}$ and $z^{*}=z$;

(3) $x^{*}=x^{-1}, y^{*}=y^{-1}$ and $z^{*}=z^{-1}$.

We have two possibilities: either $z$ is transcendental over the prime field $P$ of $k$ or $z$ is algebraic over $P$. In this last case, $P=\mathbb{Q}$, because if $z$ where algebraic over a finite field, it would have finite order, but it is contained in the torsion-free group $G$.

Suppose $z$ is transcendental over $P$. In this case, it has been shown in the proof of [7, Theorem 1.3] that the group algebra $P[\Gamma]$ embeds into $D$, and we can follow the steps trodden in the past theorem. Let $D^{\prime}$ be the subdivision ring of $D$ generated by $P[\Gamma]$ and let $\psi$ be the usual specialization from $D^{\prime}$ to the quaternion algebra $\mathcal{H}$ satisfying $\psi(x)=\mathbf{i}, \psi(y)=\mathbf{j}$ and $\psi(z)=-1$. We consider each of the three possibilities for $*$ on the generators separately: 
(1) $x^{*}=x, y^{*}=y$ and $z^{*}=z^{-1}$. Let $u=1+x$ and $v=1+y$. Since $x, y \in G$, both $s=u y^{-1} u^{-1} y$ and $t=v x^{-1} v^{-1} x$ belong to $N \cap D^{\prime}$, and we have $\psi(s)=(1-a)^{-1}(1+\mathbf{i})^{2}$, $\psi(t)=(1-b)^{-1}(1+\mathbf{j})^{2}$. Since $N$ is invariant under $*$, it follows that $s s^{*}, t t^{*} \in N$. Now, by [6. Proposition 16], it follows that $\psi\left(s s^{*}\right)=(1-a)^{-2}(1+\mathbf{i})^{4}$ and $\psi\left(t t^{*}\right)=(1-b)^{-2}(1+\mathbf{j})^{4}$ generate a free subgroup; so, $\left\{s s^{*}, t t^{*}\right\}$ is a free symmetric pair.

(2) $x^{*}=x, y^{*}=y^{-1}$ and $z^{*}=z$. Let $u=1+x$ and $v=1+y+y^{-1}$. We have $\psi(u)=1+\mathbf{i}$ and $\psi(v)=1+\beta \mathbf{j}$, where $\beta=1+b^{-1}$. Defining $s=u y u^{-1} y^{-1}$ and $t=v x v^{-1} x^{-1}$, we get $\psi(s)=(1-a)^{-1} b^{-1}(1+\mathbf{i}) \mathbf{j}(1-\mathbf{i}) \mathbf{j}$ and, so, $\psi\left(s s^{*}\right)=(1-a)^{-2}(1+\mathbf{i})^{4}$. Similarly $\psi\left(t t^{*}\right)=\left(1-\beta^{2} b\right)^{-2}(1+\beta \mathbf{j})^{4}$. By [6, Theorem 2], $\psi\left(s s^{*}\right)$ and $\psi\left(t t^{*}\right)$ generate a free subgroup. So the same is true of the symmetric elements $s s^{*}$ and $t t^{*}$ in $N$.

(3) $x^{*}=x^{-1}, y^{*}=y^{-1}$ and $z^{*}=z^{-1}$. Let $u=1+x+x^{-1}$ and $v=1+y+y^{-1}$, and define $s=y u y^{-1} u^{-1}$ and $t=x v x^{-1} v^{-1}$. Using the abbreviations $\alpha=1+a^{-1}$ and $\beta=1-b^{-1}$, we have

$$
\psi\left(s s^{*}\right)=\frac{(1-\alpha \mathbf{i})^{4}}{\left(1-\alpha^{2} a\right)^{2}} \quad \text { and } \quad \psi\left(t t^{*}\right)=\frac{(1-\beta \mathbf{j})^{4}}{\left(1-\beta^{2} b\right)^{2}} .
$$

From [6, Theorem 2], it follows that $\left\{\psi\left(s s^{*}\right), \psi\left(t t^{*}\right)\right\}$ is a free pair, and consequently $\left\{s s^{*}, t t^{*}\right\}$ is a free symmetric pair in $N$.

Suppose $z$ is algebraic over $\mathbb{Q}$. Here, the construction in the proof of [7, Theorem 1.3] provides a specialization $\psi$ from $D^{\prime}$ to $\mathcal{H}$ such that $\psi\left(x^{2^{n}}\right)=\mathbf{i}, \psi(y)=\mathbf{j}$ and $\psi\left(z^{2^{n}}\right)=-1$, for a suitable positive integer $n$. Substituting $x$ by $x^{2^{n}}$ in the formulas occurring in the case of $z$ transcendental over $P$, considered above, and acting accordingly, we obtain our free symmetric pairs.

\section{RESiduALly TORSION-FREE NILPOTENT GROUPS}

In this section we show how to extend the results in the previous section to residually torsionfree nilpotent groups through an argument, used previously in [2] and [19, involving the MalcevNeumann series ring defined by an orderable group.

3.1. Preliminaries. The following definitions and comments are from [18, Chapter 1].

Let $R$ be a ring and let $G$ be a group. A crossed product of $G$ over $R$ is an associative ring $R[\bar{G} ; \sigma, \tau]$ which contains $R$ as a subring and it is a free left $R$-module with $R$-basis the set $\bar{G}$, a copy of $G$. Thus each element of $R[\bar{G} ; \sigma, \tau]$ is uniquely expressed as a finite sum $\sum_{x \in G} r_{x} \bar{x}$, with $r_{x} \in R$. Addition is component-wise and multiplication is determined by the two rules below. For $x, y \in G$ we have a twisting

$$
\bar{x} \bar{y}=\tau(x, y) \overline{x y},
$$

where $\tau$ is a map from $G \times G$ to the group of units $U(R)$ of $R$. Furthermore, for $x \in G$ and $r \in R$, we have an action

$$
\bar{x} r=r^{\sigma(x)} \bar{x}
$$

where $\sigma: G \rightarrow \operatorname{Aut}(R)$ and $r^{\sigma(x)}$ denotes the image of $r$ by $\sigma(x)$.

If $d: G \rightarrow U(R)$ assigns to each element $x \in G$ a unit $d_{x}$ of $R$, then $\widetilde{G}=\left\{\widetilde{x}=d_{x} \bar{x}: x \in G\right\}$ is another $R$-basis for $R[\bar{G} ; \sigma, \tau]$. Moreover there exist maps $\sigma^{\prime}: G \rightarrow \operatorname{Aut}(R)$ and $\tau^{\prime}: G \times G \rightarrow U(R)$ such that $R[\bar{G} ; \sigma, \tau]=R\left[\widetilde{G} ; \sigma^{\prime}, \tau^{\prime}\right]$. We call this a diagonal change of basis. Via a diagonal change of basis, if necessary, we will assume that $1_{R[\bar{G} ; \sigma, \tau]}=\overline{1}$. The embedding of $R$ into $R[\bar{G} ; \sigma, \tau]$ is then given by $r \mapsto r \overline{1}$. Note that $\bar{G}$ acts on both $R[\bar{G} ; \sigma, \tau]$ and $R$ by conjugation and that for $x \in G$ and $r \in R$, we have $\bar{x} r \bar{x}^{-1}=r^{\sigma(x)}$.

If there is no action or twisting, that is, if $\sigma(x)$ is the identity map and $\tau(x, y)=1$ for all $x, y \in G$, then $R[\bar{G} ; \sigma, \tau]=R[G]$, the usual group ring. 
If $N$ is a subgroup of $G$, the crossed product $R\left[\bar{N} ; \sigma_{\mid N}, \tau_{\mid N \times N}\right]$ embeds into $R[\bar{G} ; \sigma, \tau]$, and we will denote it by $R[\bar{N} ; \sigma, \tau]$.

Suppose that $N$ is a normal subgroup of $G$ and let $S=R[\bar{N} ; \sigma, \tau]$. Then $R[\bar{G} ; \sigma, \tau]$ can be seen as a crossed product of $G / N$ over $S$. More precisely $R[\bar{G} ; \sigma, \tau]=S[\overline{G / N} ; \widetilde{\sigma}, \widetilde{\tau}]$, for appropriate $\widetilde{\sigma}$ and $\widetilde{\tau}$. Below we will show in detail how this is done for a group ring $R[G]$.

A group $G$ is orderable if there exists a total order $<$ in $G$ such that, for all $x, y, z \in G$,

$$
x<y \quad \text { implies that } z x<z y \text { and } x z<y z \text {. }
$$

In this event we say that $(G,<)$ is an ordered group. For further details on ordered groups the reader is referred to any monograph on the subject such as [3].

Let $R$ be a ring and let $(G,<)$ be an ordered group. Suppose that $R[\bar{G} ; \sigma, \tau]$ is a crossed product of $G$ over $R$. We define a new ring, denoted $R((\bar{G} ; \sigma, \tau,<))$ and called Malcev-Neumann series ring, in which $R[\bar{G} ; \sigma, \tau]$ embeds. As a set,

$$
R((\bar{G} ; \sigma, \tau,<))=\left\{f=\sum_{x \in G} a_{x} \bar{x}: a_{x} \in R, \operatorname{supp}(f) \text { is well-ordered }\right\}
$$

where $\operatorname{supp}(f)=\left\{x \in G \mid a_{x} \neq 0\right\}$.

Addition and multiplication are defined extending the ones in $R[\bar{G} ; \sigma, \tau]$. That is, given $f=$ $\sum_{x \in G} a_{x} \bar{x}$ and $g=\sum_{x \in G} b_{x} \bar{x}$, elements of $R((\bar{G} ; \sigma, \tau,<))$, one has

$$
f+g=\sum_{x \in G}\left(a_{x}+b_{x}\right) \bar{x} \quad \text { and } \quad f g=\sum_{x \in G}\left(\sum_{y z=x} a_{y} b_{z}^{\sigma(y)} \tau(y, z)\right) \bar{x} .
$$

When the crossed product is a group ring $R[G]$, its Malcev-Neumann series ring will be denoted by $R((G ;<))$.

In general, for any ring $R$ and $f \in R((\bar{G} ; \sigma, \tau,<))$, if the coefficient of min supp $f$ is an invertible element of $R$, then $f$ is invertible. Hence, if $R$ is a division $\operatorname{ring}$, then $R((\bar{G} ; \sigma, \tau,<))$ will be a division ring (as proved in [15] and [16]). If $R$ is a domain, then $R((\bar{G} ; \sigma, \tau,<))$ is a domain, and $f \in R((\bar{G} ; \sigma, \tau,<))$ is invertible if and only if the coefficient of $\min \operatorname{supp} f$ is invertible in $R$.

If $K$ is a division ring, the division subring of $K((\bar{G} ; \sigma, \tau,<))$ generated by $K[\bar{G} ; \sigma, \tau]$ will be called the Malcev-Neumann division ring of fractions of $K[\bar{G} ; \sigma, \tau]$ and denoted by $K(\bar{G} ; \sigma, \tau)$. It is important to observe the following. For a subgroup $H$ of $G, K((\bar{H} ; \sigma, \tau))$ and $K(\bar{H} ; \sigma, \tau)$ can be regarded as division subrings of $K((\bar{G} ; \sigma, \tau))$ and $K(\bar{G} ; \sigma, \tau)$, respectively, in the natural way. In the case of the group ring $K[G]$, the Malcev-Neumann division ring of fractions is denoted by $K(G)$. We remark that $K(\bar{G} ; \sigma, \tau)$ does not depend on the order $<$ of $G$, see [10]. When the crossed product $K[\bar{G} ; \sigma, \tau]$ is an Ore domain, then $K(G ; \sigma, \tau)$ is the Ore ring of quotients of $K[\bar{G} ; \sigma, \tau]$.

3.2. The series argument. Let $k$ be a field, $G$ a group and $N$ a normal subgroup of $G$. Then the group ring $k[G]$ can be regarded as a crossed product $k[N][\overline{G / N} ; \sigma, \tau]$ of the group $G / N$ over the ring $k[N]$. More precisely, for each $1 \neq \alpha \in G / N$, let $x_{\alpha} \in G$ be a fixed representative of the class $\alpha$. For the class $1 \in G / N$ choose $x_{1}=1 \in G$. Set $\bar{\alpha}=x_{\alpha}$ for each $\alpha \in G / N$. Then $G$ can be written as a disjoint union $G=\bigcup\{N \bar{\alpha}: \alpha \in G / N\}$. Define $\overline{G / N}=\{\bar{\alpha}: \alpha \in G / N\} \subseteq G$. This shows that $k[G]=\bigoplus_{\alpha \in G / N} k[N] \bar{\alpha}$. Therefore $\overline{G / N}$ is a $k[N]$-basis for $k[G]$. Moreover, we have maps

$$
\sigma: G / N \longrightarrow \operatorname{Aut}(k[N]) \text { and }{ }_{8} \tau: G / N \times G / N \longrightarrow U(k[N])
$$


such that $\sigma(\alpha)(y)=x_{\alpha} y x_{\alpha}^{-1}$, for all $\alpha \in G / N$ and $y \in k[N]$, and $\tau(\alpha, \beta)=n_{\alpha \beta}$ is the unique element in $N$ such that $n_{\alpha \beta} x_{\alpha \beta}=x_{\alpha} x_{\beta}$, for all $\alpha, \beta \in G / N$. Then,

$$
\bar{\alpha}\left(\sum_{n \in N} a_{n} n\right)=\left(\sum_{n \in N} a_{n} n\right)^{\sigma(\alpha)} \bar{\alpha} \quad \text { and } \quad \bar{\alpha} \bar{\beta}=\tau(\alpha, \beta) \overline{\alpha \beta},
$$

as desired.

Notice that the assumption $x_{1}=1$ implies that $k[N]$ embeds in $k[N][\overline{G / N} ; \sigma, \tau]$ via an $\mapsto$ an $\cdot 1$.

The next result shows that writing a group algebra as a crossed product over a quotient group allows the construction of an algebra homomorphism onto the Malcev-Neumann series ring of the quotient group, in case it is orderable. This will be used in order to pull back results known to be valid over "simpler" groups to more general situations.

Proposition 3.1. Let $k$ be a field, let $G$ be a group and let $N$ be a normal subgroup of $G$ such that $G / N$ is orderable. Suppose that $<$ is a total order in $G / N$ such that $(G / N,<)$ is an ordered group. Let $\left\{x_{\alpha}\right\}_{\alpha \in G / N}$ be a left transversal of $N$ in $G$ with $x_{1}=1$ and regard the group ring $k[G]$ as a crossed product $k[N][\overline{G / N} ; \sigma, \tau]$. Let $\varepsilon_{N}: k[N] \rightarrow k$ denote the usual augmentation map. Then the $\operatorname{map} \Phi_{N}: k[N]((\overline{G / N} ; \sigma, \tau,<)) \rightarrow k((G / N ;<))$ defined by

$$
\Phi_{N}\left(\sum_{\alpha \in G / N} f_{\alpha} \bar{\alpha}\right)=\sum_{\alpha \in G / N} \varepsilon_{N}\left(f_{\alpha}\right) \alpha,
$$

where $f_{\alpha} \in k[N]$, for each $\alpha \in G / N$, is a k-algebra homomorphism.

Proof. The map $\Phi_{N}$ is well defined by the definition of the Malcev-Neumann series rings. Let $f, g \in K[N]((\overline{G / N} ; \sigma, \tau ;<))$, say $f=\sum_{\alpha \in G / N} f_{\alpha} \bar{\alpha}$ and $g=\sum_{\alpha \in G / N} g_{\alpha} \bar{\alpha}, f_{\alpha}, g_{\alpha} \in k[N]$, for each $\alpha \in G / N$. Then, clearly, $\Phi_{N}(f+g)=\Phi_{N}(f)+\Phi_{N}(g)$ and

$$
\begin{aligned}
\Phi_{N}(f g) & =\Phi_{N}\left(\left(\sum_{\alpha \in G / N} f_{\alpha} \bar{\alpha}\right)\left(\sum_{\beta \in G / N} g_{\beta} \bar{\beta}\right)\right) \\
& =\Phi_{N}\left(\sum_{\gamma \in G / N}\left(\sum_{\alpha \beta=\gamma} f_{\alpha} g_{\beta}^{\sigma(\alpha)} \tau(\alpha, \beta)\right) \bar{\gamma}\right) \\
& =\sum_{\gamma \in G / N} \varepsilon_{N}\left(\sum_{\alpha \beta=\gamma} f_{\alpha} g_{\beta}^{\sigma(\alpha)} \tau(\alpha, \beta)\right) \gamma .
\end{aligned}
$$

Since $\tau(\alpha, \beta)=n_{\alpha \beta}$ is the unique element in $N$ such that $x_{\alpha} x_{\beta}=n_{\alpha \beta} x_{\alpha \beta}$, it follows that $\varepsilon_{N}(\tau(\alpha, \beta))=1$. Now, given $g_{\beta}=\sum_{n \in N} b_{\beta n} n$, with $b_{\beta n} \in k$ and $n \in N$, then

$$
g_{\beta}^{\sigma(\alpha)}=x_{\alpha}\left(\sum_{n \in N} b_{\beta n} n\right) x_{\alpha}^{-1}=\sum_{n \in N} b_{\beta n} x_{\alpha} n x_{\alpha}^{-1} .
$$

Hence, $\varepsilon_{N}\left(g_{\beta}\right)=\varepsilon_{N}\left(g_{\beta}^{\sigma(\alpha)}\right)$ and, therefore, $\varepsilon_{N}\left(\sum_{\alpha \beta=\gamma} f_{\alpha} g_{\beta}^{\sigma(\alpha)} \tau(\alpha, \beta)\right)=\left(\sum_{\alpha \beta=\gamma} \varepsilon_{N}\left(f_{\alpha}\right) \varepsilon_{N}\left(g_{\beta}\right)\right)$. So,

$$
\begin{aligned}
\Phi_{N}(f g) & =\sum_{\gamma \in G / N}\left(\sum_{\alpha \beta=\gamma} \varepsilon_{N}\left(f_{\alpha}\right) \varepsilon_{N}\left(g_{\beta}\right)\right) \gamma \\
& =\left(\sum_{\alpha \in G / N} \varepsilon_{N}\left(f_{\alpha}\right) \alpha\right)\left(\sum_{\beta \in G / N} \varepsilon_{N}\left(g_{\beta}\right) \beta\right) \\
& =\Phi(f) \Phi(g) .
\end{aligned}
$$

We now introduce a notion that applies to residually torsion-free nilpotent groups. 
Definition 3.2. Let $k$ be a field, let $G$ be a group and suppose that the group $\operatorname{ring} k[G]$ embeds in a division ring $D$. If $H$ is a subgroup of $G$, denote by $D_{H}$ the subdivision ring of $D$ generated by $k[H]$. Let $N$ be a normal subgroup of $G$. We say that the embedding $k[G] \hookrightarrow D$ is a $\operatorname{good}$ embedding for $k[N]$ if the following conditions are satisfied:

(i) the group $G / N$ is orderable;

(ii) for all division rings $F$, any crossed product $F[\overline{G / N} ; \sigma, \tau]$ is an Ore domain;

(iii) there exists a left transversal $\left\{x_{\alpha}\right\}_{\alpha \in G / N}$ of $N$ in $G$ which is left $D_{N}$-linearly independent.

In the context of Definition 3.2, it is not difficult to prove that condition (iii) implies that any left transversal $\left\{x_{\alpha}\right\}_{\alpha \in G / N}$ of $N$ in $G$ is left $D_{N}$-linearly independent. Also, condition (iii) implies that the subring of $D$ generated by $D_{N}$ and $\left\{x_{\alpha}\right\}_{\alpha \in G / N}$ is a crossed product $D_{N}[\overline{G / N} ; \sigma, \tau]$.

Before giving examples of good embeddings, let us recall some known results. Let $W$ be a torsion-free nilpotent group. By, for example, [17, Lemma 13.1.6], $W$ is orderable. Moreover, any crossed product $F[\bar{W} ; \sigma, \tau]$, with $F$ a division ring, is an Ore domain by [18, Corollary 37.11], for example. With this results in mind, we proceed to present the main examples of good embeddings for our purposes.

Example 1. Let $k$ be a field and let $G$ be a group such that the group algebra $k[G]$ is an Ore domain. Let $D$ be the Ore division ring of fractions of $k[G]$. Then $k[G] \hookrightarrow D$ is a good embedding for any normal subgroup $N$ of $G$ such that $G / N$ is torsion-free nilpotent. This follows from the fact that $k[N]$ is an Ore domain for any subgroup $N$ of $G$. Hence $D_{N}$ is the Ore division ring of fractions of $k[N]$, and the elements of $D_{N}$ are left fractions $p^{-1} q$ with $p, q \in k[N], p \neq 0$. Using the common denominator property, it is not difficult to prove that any left transversal of $N$ in $G$ is left $D_{N}$-linearly independent (because it is left $k[N]$-linearly independent).

Example 2. Let $k$ be a field, let $(G,<)$ be an ordered group and let $k(G)$ denote the MalcevNeumann division ring of fractions of the group algebra $k[G]$. Then $k[G] \hookrightarrow k(G)$ is a good embedding for any normal subgroup $N$ of $G$ such that $G / N$ is torsion-free nilpotent. For condition (iii), let $\left\{x_{\alpha}\right\}_{\alpha \in G / N}$ be a left transversal of $N$ in $G$. For any $n, n^{\prime} \in N$ and $\alpha, \alpha^{\prime} \in G / N, n x_{\alpha}=n^{\prime} x_{\alpha^{\prime}}$ if, and only if, $n=n^{\prime}$ and $x_{\alpha}=x_{\alpha^{\prime}}$. Since $k(N)$, the division ring generated by $k[N]$ inside $k(G)$, is contained in $k((N ;<)) \subseteq k((G ;<))$, it is not difficult to prove that the elements of the left transversal are left linearly independent over $k((N ;<))$ and, hence, over $k(N)$.

Given a field $k$, a group $G$ and a division ring $D$ such that $k[G]$ embeds in $D$, let $N$ be a normal subgroup of $G$ such that the embedding $k[G] \hookrightarrow D$ is good for $k[N]$. Suppose that $G / N$ is endowed with a total order such that $(G / N,<)$ is an ordered group. By condition (ii), $D_{N}[\overline{G / N} ; \sigma, \tau]$ is an Ore domain embedded in $D_{G}$. By the universal property of the Ore localization, $D_{G}$ is the Ore division ring of quotients of $D_{N}[\overline{G / N} ; \sigma, \tau]$. But $D_{N}[\overline{G / N} ; \sigma, \tau]$ also embeds in the division ring $D_{N}((G / N ; \sigma, \tau,<))$ and, again, by the universal property of the Ore localization $D_{N}(G / N ; \sigma, \tau)$ is the Ore division ring of quotients of $D_{N}[\overline{G / N} ; \sigma, \tau]$. Therefore $D_{G}$ can be identified with $D_{N}(G / N ; \sigma, \tau)$ inside the Malcev-Neumann series ring $D_{N}((G / N ; \sigma, \tau,<))$.

Next, we show that good embeddings provide an appropriate setting for pulling back free pairs from division rings of fractions of torsion-free nilpotent group algebras to more general division rings.

Theorem 3.3. Let $G$ be a group with an involution $*$ and let $N$ be a $*$-invariant normal subgroup of $G$ such that $G / N$ is a nonabelian torsion-free nilpotent group. Let $k$ be a field of characteristic different from 2 and let $k[G] \hookrightarrow D$ be a good embedding for $k[N]$ of the group algebra $k[G]$ in the division ring $D$. Suppose that the induced $k$-involution $*$ on $k[G]$ can be extended to a $k$-involution in $D$. Then $D_{G}$ contains a free symmetric pair with respect to $*$. 
Proof. First observe that $*$ defines a $k$-involution on $k[N]$ by restriction. It also induces an involution $*$ on the quotient group $G / N$, and, therefore, a $k$-involution on $k[G / N]$. Moreover this can be extended to an involution $*$ on $k(G / N)$, because $k[G / N]$ is an Ore domain.

Let $\left\{x_{\alpha}\right\}_{\alpha \in G / N}$ be a left transversal of $N$ in $G$ with $x_{1}=1$. Regard the group algebra $k[G]$ as a crossed product $k[N][\overline{G / N} ; \sigma, \tau]$ and let $\varepsilon_{N}: k[N] \rightarrow k$ denote the augmentation map. Observe that, for each $\alpha \in G / N$, there exists a unique $n_{\alpha} \in N$ such that $\bar{\alpha}^{*}=x_{\alpha}^{*}=n_{\alpha} x_{\alpha^{*}}=n_{\alpha} \overline{\alpha^{*}}$. Take $\sum_{\alpha \in G / N} f_{\alpha} \bar{\alpha} \in k[G]$ with $f_{\alpha} \in k[N]$. Then

$$
\left(\sum_{\alpha \in G / N} f_{\alpha} \bar{\alpha}\right)^{*}=\sum_{\alpha \in G / N} \bar{\alpha}^{*} f_{\alpha}^{*}=\sum_{\alpha \in G / N} n_{\alpha}\left(f_{\alpha}^{*}\right)^{\sigma\left(\alpha^{*}\right)} \overline{\alpha^{*}} .
$$

So, if $f_{\alpha} \in k$, for all $\alpha \in G / N$, we have

$$
\begin{aligned}
\left(\sum_{\alpha \in G / N} f_{\alpha} \bar{\alpha}\right)^{*} & =\sum_{\alpha \in G / N} n_{\alpha}\left(f_{\alpha}^{*}\right)^{\sigma\left(\alpha^{*}\right) \overline{\alpha^{*}}}=\sum_{\alpha \in G / N} n_{\alpha} f_{\alpha} \overline{\alpha^{*}} \\
& =\sum_{\alpha \in G / N} f_{\alpha} n_{\alpha} \overline{\alpha^{*}} .
\end{aligned}
$$

Consider, now, the homomorphism $\Phi_{N}: k[N]((\overline{G / N} ; \sigma, \tau,<)) \rightarrow k((G / N ;<))$, given in Proposition 3.1, defined by

$$
\Phi_{N}\left(\sum_{\alpha \in G / N} f_{\alpha} \bar{\alpha}\right)=\sum_{\alpha \in G / N} \varepsilon_{N}\left(f_{\alpha}\right) \alpha,
$$

where $f_{\alpha} \in k[N]$, for each $\alpha \in G / N$.

Suppose that $A$ is a symmetric nonzero element in $k(G / N)$ with respect to *. Then $A=p_{A} q_{A}^{-1}$ for some $p_{A}, q_{A} \in k[G / N] \backslash\{0\}$. Write $p_{A}=\sum_{\alpha \in G / N} a_{\alpha} \alpha$ and $q_{A}=\sum_{\alpha \in G / N} b_{\alpha} \alpha$, with $a_{\alpha}, b_{\alpha} \in k$ for each $\alpha \in G / N$. Define $P_{A}=\sum_{\alpha \in G / N} a_{\alpha} \bar{\alpha}$ and $Q_{A}=\sum_{\alpha \in G / N} b_{\alpha} \bar{\alpha} \in k[G]$. Note that $P_{A}$ and $Q_{A}$ are invertible elements in $k[N]((G / N ; \sigma, \tau,<))$ because the least element in the support of $P_{A}$ and $Q_{A}$ is invertible in $k[N]$ since it belongs to $k \backslash\{0\}$.

Consider also $P_{A}^{*}$ and $Q_{A}^{*}$ as elements of $k[N]((G / N ; \sigma, \tau,<))$. By (†), the least elements in the supports of $P_{A}^{*}$ and $Q_{A}^{*}$ are of the form $a_{\alpha} n_{\alpha}$ for some $n_{\alpha} \in N$ and nonzero $a_{\alpha} \in k$. Hence $P_{A}^{*}$ and $Q_{A}^{*}$ are also invertible elements in $k[N]((G / N ; \sigma, \tau,<))$.

We have

$$
\begin{aligned}
\Phi_{N}\left(P_{A}\right) & =\Phi_{N}\left(\sum_{\alpha \in G / N} a_{\alpha} \bar{\alpha}\right)=\sum_{\alpha \in G / N} \varepsilon_{N}\left(a_{\alpha}\right) \alpha \\
& =\sum_{\alpha \in G / N} a_{\alpha} \alpha=p_{A} .
\end{aligned}
$$

and

$$
\begin{aligned}
\Phi_{N}\left(P_{A}^{*}\right) & =\Phi_{N}\left(\left(\sum_{\alpha \in G / N} a_{\alpha} \bar{\alpha}\right)^{*}\right)=\Phi_{N}\left(\sum_{\alpha \in G / N} a_{\alpha} n_{\alpha} \overline{\alpha^{*}}\right) \\
& =\sum_{\alpha \in G / N} \varepsilon\left(a_{\alpha} n_{\alpha}\right) \alpha^{*}=\sum_{\alpha \in G / N} a_{\alpha} \alpha^{*}=p_{A}^{*} .
\end{aligned}
$$

Similarly, $\Phi_{N}\left(Q_{A}\right)=q_{A}$ and $\Phi_{N}\left(Q_{A}^{*}\right)=q_{A}^{*}$. Since $\Phi_{N}$ is a ring homomorphism, it follows that $\Phi_{N}\left(Q_{A}^{-1}\right)=q_{A}^{-1}$ and $\Phi_{N}\left(\left(Q_{A}^{*}\right)^{-1}\right)=\left(q_{A}^{*}\right)^{-1}$. 
Consider now the symmetric element $X=P_{A} Q_{A}^{-1}\left(Q_{A}^{*}\right)^{-1} P_{A}^{*} \in D_{G}$. Then

$$
\begin{aligned}
\Phi_{N}(X) & =\Phi_{N}\left(P_{A} Q_{A}^{-1}\left(Q_{A}^{*}\right)^{-1} P_{A}^{*}\right) \\
& =\Phi_{N}\left(P_{A}\right) \Phi_{N}\left(Q_{A}^{-1}\right) \Phi_{N}\left(\left(Q_{A}^{*}\right)^{-1}\right) \Phi_{N}\left(P_{A}^{*}\right) \\
& =p_{A} q_{A}^{-1}\left(q_{A}^{*}\right)^{-1} p_{A}^{*} \\
& =p_{A} q_{A}^{-1}\left(p_{A} q_{A}^{-1}\right)^{*} \\
& =A A^{*} \\
& =A^{2}
\end{aligned}
$$

Since $P_{A}, P_{A}^{-1}, P_{A}^{*},\left(P_{A}^{*}\right)^{-1}, Q_{A}, Q_{A}^{-1}, Q_{A}^{*},\left(Q_{A}^{*}\right)^{-1} \in k[N]((G / N ; \sigma, \tau,<))$, if follows that $X^{-1} \in$ $k[N]((G / N ; \sigma, \tau,<))$. Thus $\Phi_{N}\left(X^{-1}\right)=A^{-2}$.

Now let $A, B$ be symmetric elements of $k(G / N)$ generating a free group, which exist by Theorem 1.3. Clearly, $A^{2}, B^{2}$ also generate a free group. By the foregoing argument, there exist $X, Y \in D_{G}$ such that $\Phi_{N}(X)=A^{2}, \Phi_{N}(Y)=B^{2}, \Phi_{N}\left(X^{-1}\right)=A^{-2}$ and $\Phi_{N}\left(Y^{-1}\right)=B^{-2}$. Therefore $X, Y$ also generate a free group.

3.3. Proof of Theorem 1.6. Here we shall apply the main result of the preceding section in order to extend Theorem 1.3 to the class of residually torsion-free nilpotent groups.

Definition 3.4. A group $G$ is residually torsion-free nilpotent if for each $g \in G$, there exists a normal subgroup $N_{g}$ of $G$ such that $g \notin N_{g}$ and $G / N_{g}$ is torsion-free nilpotent.

Let $G$ be a group. If $H$ is a subgroup of $G$, we denote by $\sqrt{H}$ the subset of $G$ defined by

$$
\sqrt{H}=\left\{x \in G: x^{n} \in H \text {, for some } n \geq 1\right\} .
$$

For the next result, given a group $G$ we shall denote the $n$-th term in its lower central series by $\gamma_{n}(G)$. That is, we set $\gamma_{1}(G)=G$ and, for $n \geq 1$, define $\gamma_{n+1}(G)=\left[\gamma_{n}(G), G\right]$.

Lemma 3.5. Let $G$ be a residually torsion-free nilpotent group. Then the following hold true.

(1) For each $n \geq 1, \sqrt{\gamma_{n}(G)}$ is a normal subgroup of $G$ such that $G / \sqrt{\gamma_{n}(G)}$ is torsion-free nilpotent.

(2) The chain of normal subgroups of $G$

$$
G=\sqrt{\gamma_{1}(G)} \supseteq \sqrt{\gamma_{2}(G)} \supseteq \cdots \supseteq \sqrt{\gamma_{n}(G)} \supseteq \cdots
$$

satisfies

(a) $\left[G, \sqrt{\gamma_{n}(G)} \subseteq \sqrt{\gamma_{n+1}(G)}\right.$, for all $n \geq 1$, and

(b) $\sqrt{\gamma_{n}(G)} / \sqrt{\gamma_{n+1}(G)}$ is a torsion-free abelian group, for all $n \geq 1$.

(3) $\bigcap_{n \geq 1} \sqrt{\gamma_{n}(G)}=\{1\}$.

(4) $G$ is an orderable group.

Proof. (11) and (2) follow from [17, Lemma 11.1.8]. For (3), given $g \in G$, since $G / N_{g}$ is nilpotent, there exists $n_{g} \geq 1$ such that $\gamma_{n_{g}}(G) \subseteq N_{g}$. Moreover, since $G / N_{g}$ is a torsion-free group,

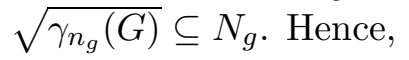

$$
\bigcap_{n \geq 1} \gamma_{n}(G) \subseteq \bigcap_{n \geq 1} \sqrt{\gamma_{n}(G)} \subseteq \bigcap_{g \in G} \sqrt{\gamma_{n_{g}}(G)} \subseteq \bigcap_{g \in G} N_{g}=\{1\} .
$$

Now, (4) follows from (10), (22), (3), in view of [3, Theorem IV.6].

We are ready to present a proof of Theorem 1.6. 
Proof of Theorem [1.6. The $k$-involution on the group algebra $k[G]$ induced by the involution $*$ on $G$ extends uniquely to a $k$-involution on the Malcev-Neumann division ring of fractions $k(G)$ of $k[G]$ (see [2, Theorem 2.9]). Let $n \geq 2$ be fixed. Since $G$ is not abelian, $G / \sqrt{\gamma_{n}(G)}$ is a nonabelian torsion-free nilpotent group. Apply Theorem 3.3 with $N=\sqrt{\gamma_{n}(G)}$.

It should be remarked that the argument used in the proofs of Theorems 3.3 and 1.6 can also be used to prove the existence of symmetric free group algebras. More precisely, under the hypothesis of Theorem 3.3. it can be shown that if the characteristic of $k$ is zero, then there exist symmetric elements $X, Y \in D_{G}$ such that the $k$-subalgebra of $D_{G}$ generated by $\left\{X, X^{-1}, Y, Y^{-1}\right\}$ is the free group $k$-algebra generated by $\{X, Y\}$. For a proof, the same argument used in the proof of Theorem 3.3 shows that if $A$ and $B$ are symmetric elements in $k(G / N)$ generating a free group algebra, then $X$ and $Y$ will be symmetric elements in $D_{G}$ generating a free group algebra. That $k(G / N)$ contains such a pair follows from [20. Similarly, one can use this version of Theorem 3.3 to prove that if $k$ has characteristic zero and $G$ is a nonabelian residually torsion-free nilpotent group with an involution $*$, then the Malcev-Neumann division ring of fractions $k(G)$ of $k[G]$ contains a free group $k$-algebra generated by symmetric elements with respect to the $k$-involution on $k(G)$ induced by $*$.

\section{REFERENCES}

[1] V. O. Ferreira and J. Z. Gonçalves, Free symmetric and unitary pairs in division rings infinite-dimensional over their centers, Israel Journal of Mathematics 210 (2015), no. 1, 297-321.

[2] V. O. Ferreira, J. Z. Gonçalves and J. Sánchez, Free symmetric group algebras in division rings generated by poly-orderable groups, Journal of Algebra 392 (2013), 69-84.

[3] L. Fuchs, Partially ordered algebraic systems, Pergamon Press, Oxford, 1963.

[4] J. Z. Gonçalves, Free pairs of symmetric and unitary units in normal subgroups of a division ring, Journal of Algebra and its Applications 16 (2017), no. 6, 1750108, 17pp.

[5] J. Z. Gonçalves and A. I. Lichtman, Free subgroups in division rings generated by group rings of soluble groups, International Journal of Algebra and Computation 24 (2014), no. 8, 1127-1140.

[6] J. Z. Gonçalves, A. Mandel and M. Shirvani, Free products of units in algebras. I. Quaternion algebras, Journal of Algebra 214 (1999), no. 1, 301-316.

[7] J. Z. Gonçalves and D. S. Passman, Explicit free groups in division rings, Proceedings of the American Mathematical Society 143 (2015), no. 2, 459-468.

[8] J. Z. Gonçalves and M. Shirvani, Free symmetric and unitary pairs in central simple algebras with involution, Groups, rings and algebras, Contemporary Mathematics 420, American Mathematical Society, Providence, RI, 2006, pp. 121-139.

[9] J. Z. Gonçalves and M. Shirvani, A survey on free objects in division rings and in division rings with an involution, Communications in Algebra 40 (2012), no. 5, 1704-1723.

[10] I. Hughes, Division rings of fractions for group rings, Communications on Pure and Applied Mathematics 23 (1970), 181-188.

[11] M. I. Kargapolov and Ju. I. Merzljakov, Fundamentals of the theory of groups, Springer-Verlag, New York-Berlin, 1979.

[12] J. C. Lennox and D. J. S. Robinson, The theory of infinite soluble groups, Clarendon Press, Oxford, 2004.

[13] A. I. Lichtman, On subgroups of the multiplicative group of skew fields, Proceedings of the American Mathematical Society 63 (1977), no. 1, 15-16.

[14] A. I. Lichtman, Free subgroups of normal subgroups of the multiplicative group of skew fields, Proceedings of the American Mathematical Society 71 (1978), no. 2, 174-178.

[15] A. I. Malcev, On the embedding of group algebras in division algebras (Russian), Doklady Akad. Nauk SSSR (N.S.) 60 (1948), 1499-1501.

[16] B. H. Neumann, On ordered division rings, Transactions of the American Mathematical Society 66 (1949), 202-252.

[17] D. S. Passman, The algebraic structure of group rings, Robert E. Krieger Publishing Co., Inc., Melbourne, FL, 1985.

[18] D. S. Passman, Infinite crossed products, Academic Press, Inc., Boston, MA, 1989.

[19] J. Sánchez, Free group algebras in Malcev-Neumann skew fields of fractions, Forum Mathematicum 26 (2014), no. 2, 443-466. 
[20] J. Sánchez, Free group algebras in division rings with valuation II, work in progress.

Department of Mathematics, University of São Paulo, São Paulo, SP, 05508-090, Brazil E-mail address: vofer@ime.usp.br

Department of Mathematics, University of São Paulo, São Paulo, SP, 05508-090, Brazil E-mail address: jz.goncalves@usp.br

Department of Mathematics, University of São Paulo, São Paulo, SP, 05508-090, Brazil E-mail address: jsanchez@ime.usp.br 